\title{
Solid-state cooling by stress: A perspective
}

Cite as: Appl. Phys. Lett. 116, 050501 (2020); https://doi.org/10.1063/1.5140555

Submitted: 28 November 2019 . Accepted: 18 January 2020 . Published Online: 03 February 2020

\section{Dluís Mañosa, and (iD Antoni Planes}

\section{COLLECTIONS}

F This paper was selected as Featured
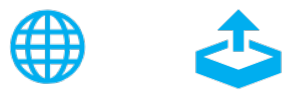

\section{ARTICLES YOU MAY BE INTERESTED IN}

Progress, challenges, and perspective on metasurfaces for ambient radio frequency energy harvesting

Applied Physics Letters 116, 060501 (2020); https://doi.org/10.1063/1.5140966

\section{Development of microLED}

Applied Physics Letters 116, 100502 (2020); https://doi.org/10.1063/1.5145201

Oxygen vacancies: The (in)visible friend of oxide electronics

Applied Physics Letters 116, 120505 (2020); https://doi.org/10.1063/1.5143309

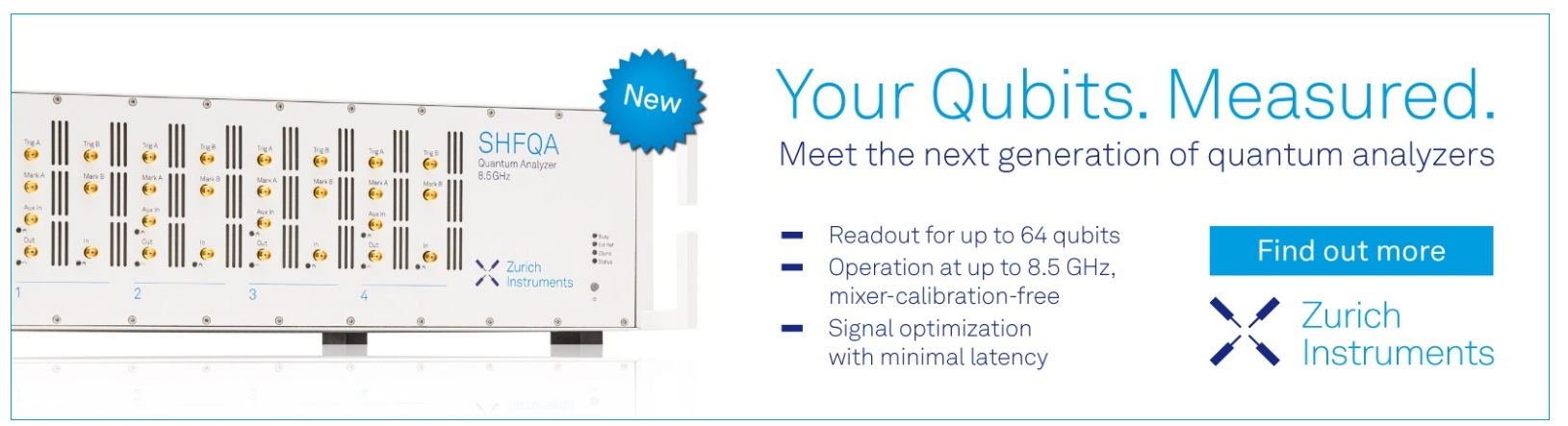




\title{
Solid-state cooling by stress: A perspective
}

\author{
Cite as: Appl. Phys. Lett. 116, 050501 (2020); doi: 10.1063/1.5140555 \\ Submitted: 28 November 2019 - Accepted: 18 January 2020 . \\ Published Online: 3 February 2020
}
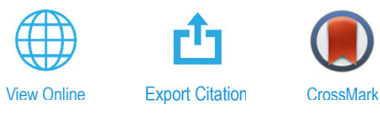

\section{Lluís Mañosa $^{a)}$ (iD and Antoni Planes ${ }^{\text {b) }}$ (iD}

\author{
AFFILIATIONS \\ Catalonia, Spain \\ a)|luis.manosa@fmc.ub.edu \\ b)antoni.planes@fmc.ub.edu
}

Departament de Física de la Matèria Condensada, Facultat de Física, Universitat de Barcelona, Martí i Franquès, 1, 08028 Barcelona,

\begin{abstract}
Materials with both giant and colossal mechanocaloric effects together with giant electrocaloric and magnetocaloric materials are expected to replace harmful fluids in more efficient and environmentally friendly refrigeration and heat pumping technologies. While mechanocaloric materials have only received attention in the last decade, they have already shown better caloric performances than their magnetic and polar counterparts. In particular, the recent discovery of colossal barocaloric and elastocaloric materials opens up bright perspectives for this class of materials. We envisage great promise in the use of mechanocaloric materials for future energy applications.
\end{abstract}

Published under license by AIP Publishing. https://doi.org/10.1063/1.5140555

Global warming poses a serious challenge to modern society. The average temperature increase in our planet is causing an increasingly greater demand for refrigeration, while, on the other hand, current refrigerators use fluids that significantly contribute to greenhouse effects. It is therefore a matter of some urgency to escape from such a vicious circle by finding alternative cooling technologies, which do not involve the use of hazardous materials and are friendly with our environment. However, according to the second law of thermodynamics, cooling requires expending energy in the form of work and thus, refrigeration is not free. We therefore need to find more efficient ways to refrigerate which have the added value of being respectful to our planet. Solid state cooling based on giant caloric effects exhibited by certain materials is seen as a good option to achieve these objectives.

Caloric effects refer to the thermal response of a solid under the application (or removal) of an external field. ${ }^{1}$ The basis of these caloric effects is the possibility of changing the thermal state of a solid by exchanging work. Actually, this idea was already pointed out by James Prescot Joule in his article On some properties of solids published in the Philosophical Transactions of the Royal Society in $1859^{2}$ where he wrote: "After finding the numerical relation between heat and work in 1843 , it immediately occurred to me to investigate various phenomena in which heat is evolved by mechanical means." Joule considered the possibility of performing mechanical work, but other kinds of work such as magnetic or electrical work can also be considered. These three ways of exchanging work are at the origin of mechano-, magneto-, and electrocaloric effects. And all of these effects have been acknowledged to be of crucial importance for the development of efficient heat pumping, refrigeration, and energy harvesting devices and a large amount of research is currently devoted to these topics. ${ }^{3}$

As previously mentioned, although caloric effects in solids were known long ago, the interest in using them for cooling close to room temperature has only just raised interest in the last few decades. The discovery of giant magnetocaloric effects close to room temperature in $\mathrm{Gd}-\mathrm{Si}-\mathrm{Ge}^{4}$ has led to research in magnetocalorics, while later discoveries of giant electrocaloric, ${ }^{5}$ elastocaloric, ${ }^{6}$ and barocaloric ${ }^{7}$ effects in several compounds have broadened the field, which now encompasses diverse caloric and multicaloric effects. ${ }^{1,8,9}$

Up until recently, most attention has been paid to the magnetocaloric effect. However, there are serious limitations that hamper the development of commercial devices using this effect. A major issue is the difficulty in creating magnetic fields high enough over a relatively large volume, capable of generating the temperature and entropy changes required for efficient refrigeration. For practical reasons, permanent magnets are normally used to generate magnetic fields. However, the fields created are rather low (2 T maximum) with correspondingly moderate values for reversible entropy and temperature changes (of the order of $10 \mathrm{~J} \mathrm{~kg}^{-1} \mathrm{~K}^{-1}$ and $\sim 5 \mathrm{~K}$, respectively). ${ }^{10}$ Furthermore, permanent magnets contain rare earth elements, which are too critical and expensive. Electrocaloric materials provide rather low temperature and entropy changes (although large theoretical values are reported, record-breaking experimental data are still modest: $\sim 6 \mathrm{~J} \mathrm{~kg}^{-1} \mathrm{~K}^{-1}$ and $\sim 5 \mathrm{~K}$ for entropy and temperature changes, respectively ${ }^{11}$ ). In addition, dielectric breakdown may occur 
at relatively low electric fields. Furthermore, materials have to be thin enough to achieve large electric fields and therefore, upscaling is difficult from a practical point of view.

In view of the above limitations, mechanocaloric effects (which encompass both elastocaloric and barocaloric effects) appear as the best alternative for both solid-state efficient and environmentally friendly refrigeration, as has been recognized by the US Department of energy in their technical report Ref. 12. As regards the mechanocaloric effect, entropy and temperature changes result from the application (or removal) of a mechanical force (stress). A priori no major difficulties are foreseen in applying relatively large forces. From the point of view of materials, mechanocaloric effects are also advantageous in relation to their magnetocaloric and electrocaloric counterparts: while magnetocaloric and electrocaloric effects are only expected to be observed in magnetic and polar materials, respectively, mechanocaloric effects are ubiquitous for any material (although the effect is only giant for certain materials). Last, but not least, the caloric performances of mechanocaloric materials (in terms of reversible adiabatic temperature and isothermal entropy changes, refrigerant capacity, etc.) outperform those reported for the best magnetocaloric and electrocaloric materials, as will be further discussed below.

Mechanocaloric effects are induced by changing any combination of the components of stress or strain tensors of a solid. Because these tensors are rank-2, even for elastically isotropic solids, two independent mechanocaloric effects associated with, for instance, shear and hydrostatic pressure must be studied. For practical reasons, instead of shear, uniaxial stress (or strain) is commonly applied, which combines both dilation and shear components. In this case, the corresponding mechanocaloric effects are denoted barocaloric (for hydrostatic pressure) and elastocaloric (for uniaxial stress). Recently, other deformation modes, associated with non-homogeneous strain, have also been considered such as the twistcaloric effect. ${ }^{13}$ The response functions quantifying barocaloric and elastocaloric effects are $\xi=(\partial \epsilon / \partial T)_{f}$, where $\epsilon$ is the deformation conjugated to the stress mode $f$. For the barocaloric effect, $\epsilon$ is the volume strain $(\omega=\Delta V / V)$ induced by hydrostatic pressure, $-p$, and for the elastocaloric effect, $\epsilon$ corresponds to the deformation $\varepsilon$ along the direction of the uniaxial stress $\sigma$. Therefore, under a change of $f$ from $f_{1}$ to $f_{2}$ the heat $Q$ exchanged by the system with an external reservoir at a temperature $T$ is

$$
Q=\Delta U-W \leq T \Delta S,
$$

with

$$
T \Delta S=T\left[S\left(T, f_{2}\right)-S\left(T, f_{1}\right)\right]=T \int_{f_{1}}^{f_{2}} \xi d f,
$$

where $\Delta U$ and $W$ are, respectively, the change of internal energy and the work exchanged with the material. It is worth noting that the equality $Q=T \Delta S$ only holds when the process is reversible, which means that optimal caloric effects will be reached under this condition. When the field is applied adiabatically, the corresponding reversible temperature change $(\Delta T)$ is determined from the condition $S\left(T+\Delta T, f_{2}\right)=S\left(T, f_{1}\right)$.

The preceding equations are interesting since they illustrate that good barocaloric and elastocaloric materials are those for which a deformation mode strongly varies with temperature. Close to room temperature, this is expected in highly deformable materials such as elastomers and it is also expected near phase transitions involving sizeable structural changes. Typically caloric effects are quantified by the field-induced adiabatic temperature $(\Delta T)$ and isothermal entropy $(\Delta S)$ changes. The reversibility of the caloric effect is also a critical factor to be taken into account in order to ensure good reproducibility of the caloric effect under field cycling.

Barocaloric effects have been reported for a wide variety of materials. The common characteristic to all these materials is the occurrence of a first-order structural transition involving a large change of volume of the unit cell. Although in many cases the transition gives rise to a change in the crystal symmetry, this is not always the case. For the majority of giant barocaloric materials, the change of volume originates from the interplay between the structure and additional degrees of freedom such as magnetic, polar, and configurational. Most magnetocaloric compounds undergo a magnetostructural transition and also exhibit large barocaloric effects (a representative list of this family of compounds can be found in Ref. 14). A different family of materials is found in spin crossover compounds in which the structural transition occurs between high spin and low spin phases. ${ }^{15}$ While spin crossover materials do not show relevant magnetocaloric properties, the spin change causes large changes of volume thereby leading to pronounced barocaloric effects. Similarly, ferroelectric and ferrielectric transitions in polar materials are also accompanied by structural transitions, with associated barocaloric effects. Examples are also listed in Ref. 14. Particularly interesting is ammonium sulfate ${ }^{16}$ because it is made from very cheap and abundant elements $\left[\left(\mathrm{NH}_{4}\right)_{2} \mathrm{SO}_{4}\right]$ and is massively produced due to its widespread agricultural use as a fertilizer. It has been recently found that in complex materials the freezing of configurational degrees of freedom at a structural phase transition involves large entropy changes with associated giant barocaloric effects. An example of these materials is hybrid organic-inorganic perovskites $^{17}$ undergoing an isostructural transition from a lowtemperature perovskite phase with ordered dicyanamide anions and tetrapropylammonium ligands toward a high-temperature phase where these organic components have larger disorder. Other examples are plastic crystals where the structural transition between ordered and plastic phases involves huge entropy changes with associated outstanding barocaloric performances. ${ }^{18-20}$ Finally, it is worth mentioning the case of AgI for which large inverse barocaloric effects occur at the superionic transition, ${ }^{21}$ thus opening up an interesting family of barocaloric materials among the solid electrolyte compounds.

Elastocaloric effects were first reported in natural rubber more than two centuries ago, ${ }^{22}$ and they have received renewed interest in recent years. ${ }^{23,24}$ The entropy changes in these materials arise from the stress-induced orientation of polymeric chains with the associated increase in the degree of order. Elastocaloric studies have also been extended to other polymeric materials. ${ }^{25}$ Adiabatic temperature and isothermal entropy changes reported for these elastomers compare well to those for magnetocaloric and electrocaloric materials, but they are lower than those corresponding to elastocaloric effects in shape memory alloys for which the giant elastocaloric effect is associated with their martensitic transition. Prototype shape memory elastocaloric materials such as Cu-based alloys, ${ }^{6,26}$ and Ti-Ni-based alloys $^{27,28}$ exhibit very large elastocaloric entropy and temperature changes. In addition, significant elastocaloric effects have also been found in different families of magnetic shape memory alloys. ${ }^{29,30}$ The magnetic $\mathrm{Fe}-\mathrm{Rh}$ alloy also exhibits elastocaloric effects ${ }^{31,32}$ associated 
with the volume change taking place at the magnetostructural transition. In several occasions, this alloy has been classified as an elastocaloric shape memory alloy but the magnetostructural transition in $\mathrm{Fe}-\mathrm{Rh}$ does not involve crystal symmetry breaking, and therefore, it cannot give rise to shape memory properties, which derive from a martensitic transition. Most magnetic shape memory alloys have the drawback of being brittle and therefore, they only support low stresses, which limits their mechanocaloric performances. However, the recently reported magnetic shape memory alloys composed of only $3 \mathrm{~d}$ elements have superior mechanical properties, with associated colossal elastocaloric properties. ${ }^{33,34}$ While the elastocaloric entropy changes in shape memory alloys are comparable to those for the best magnetocaloric materials, the reversible adiabatic temperature changes are markedly larger (in the range $10-30 \mathrm{~K}$ ). The key factor to achieve such large reversible $\Delta T$ is the extreme sensitivity of the martensitic transition to the applied uniaxial stress. Actually, such a strong sensitivity has twofold advantages. On the one hand, it is possible to drive the whole transition at moderate stresses and therefore, the caloric effect benefits from the whole transition latent heat. Furthermore, the shift in the transition temperatures is large enough to overcome the transition hysteresis and the elastocaloric effect is fully reversible. On the other hand, it enables driving elastocaloric effects using reasonable stresses at temperatures well above the material's transition temperature at zero stress thereby giving rise to a huge temperature operational window. ${ }^{35,36}$ A comparable wide operation temperature range has also recently been reported for electrocaloric oxide multilayer capacitors (MLC). ${ }^{11}$ In contrast, temperature windows in barocaloric and magnetocaloric materials are significantly narrower, about the zero-field transition temperature.

While $\Delta S$ and $\Delta T$ represent good indicators for a first screening in the search for useful caloric materials, it is also necessary to take into consideration the work $(W)$ needed to reproducibly drive these caloric effects. At present, there is no consensus on how to compare different caloric materials by an adequate figure of merit and a variety of different parameters have been proposed. For our purposes, we have selected the Coefficient of Refrigerant Performance, ${ }^{37}$ $C R P=\left|\Delta T_{\text {rev }} \Delta S / W\right|$, where $\Delta T_{\text {rev }}$ is the reversible adiabatic temperature change. In Fig. 1, we compare the best performing electrocaloric $\left(\mathrm{PbSc}_{0.5} \mathrm{Ta}_{0.5} \mathrm{O}_{3} \text { multilayer capacitors, MLC }\right)^{11}$ and magnetocaloric (Gd, La-Fe-Si and Mn-Fe-Pe-Si-based compounds) ${ }^{37}$ bulk materials, to the recently reported colossal mechanocaloric materials. For barocaloric materials, $W$ is estimated as $W \simeq-\frac{1}{2} p \Delta v$, ${ }^{38}$ with $\Delta v$ being the volume change at the phase transition, and for elastocaloric materials $W \simeq v_{0}\left(\sigma-\sigma_{0}\right) \varepsilon . v_{0}$ is the specific volume, $\sigma$ is the stress required to complete the forward martensitic transition, and $\sigma_{0}$ is the stress at which the reverse martensitic transition is finished, at a given temperature. ${ }^{39}$ Interestingly enough, by tailoring the value of the prestress $\sigma_{0}$ it is possible to achieve large elastocaloric effects over a broad temperature window which can exceed $100 \mathrm{~K} .{ }^{35}$ For electrocaloric and magnetocaloric materials, all data are from the literature. ${ }^{11,37}$

As shown in Fig. 1, the majority of caloric materials feature isothermal entropy changes well below $100 \mathrm{~J} \mathrm{~kg}^{-1} \mathrm{~K}^{-1}$ (notice that although data for magnetocaloric materials correspond to $1 \mathrm{~T}$, larger magnetic fields do not yield significantly larger values). A noteworthy exception is plastic crystals for which pressure-induced entropy changes compare to those of refrigerants used commercially (for instance R134a) in vapor compression technology. ${ }^{19,20}$ Plastic crystals

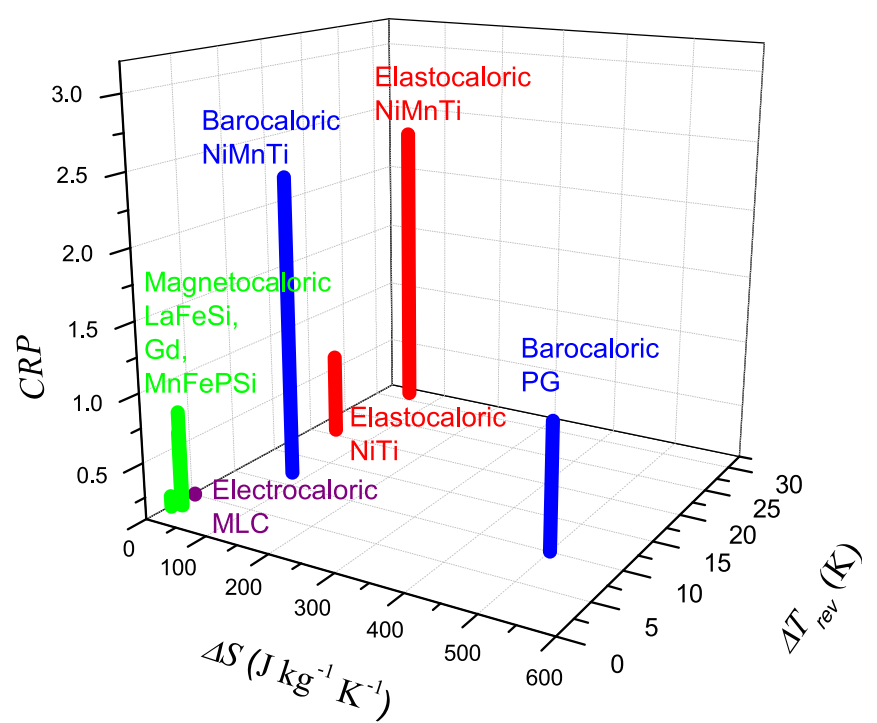

FIG. 1. Coefficient of refrigerant performance as a function of isothermal entropy and reversible adiabatic temperature changes for the best performing magnetocaloric (green), ${ }^{37}$ electrocaloric (purple), ${ }^{11}$ elastocaloric (red), ${ }^{28,33}$ and barocaloric (blue) $)^{20,34}$ bulk materials.

contain highly anisotropic organic molecules which are orientationally disordered in the high-temperature (plastic) phase and become ordered in the low-temperature phase. The freezing of this orientational disorder brings about a huge decrease in the configurational entropy. This behavior has some analogies to the freezing of magnetic moment orientations at a magnetic transition. However, the number of possible molecule orientations in plastic crystals (in the range $\sim 60$ ) is much larger than the corresponding number of spin orientations in high magnetic moment materials. Furthermore, the high compressibility of plastic crystals gives rise to an additional entropy contribution solely associated with structural degrees of freedom that also contributes to the colossal barocaloric entropy change. ${ }^{20}$ For the reported plastic crystals, the phase transition occurs with relatively large hysteresis which requires applying large pressures to achieve reversible barocaloric effects. In addition, the operational temperature window is still too narrow. There is, however, great hope that these limitations can be overcome by suitable optimization of this recent class of barocaloric materials.

Up until recently, elastocaloric studies focused to a large extent on well-known shape memory materials, among which the prototype Ti-Ni exhibited the largest elastocaloric effect. Although a decrease in performances occurs during cycling, trained $\mathrm{Ti}-\mathrm{Ni}$ samples still maintain both very large and reproducible adiabatic temperature changes of the order $\sim 20 \mathrm{~K}^{28}$ The recent discovery of a Ni-Mn-Ti Heusler shape memory alloy, composed of only $3 \mathrm{~d}$ elements, has changed this paradigm. Using ab initio calculations, the alloy has been designed to have optimal mechanical properties, weak magnetism, and large volume change at the martensitic transition, which gives rise to a large transition entropy change and a strong dependence of the transition temperature on applied stress. These properties lead to a colossal elastocaloric effect and reversible adiabatic temperature changes larger than $30 \mathrm{~K}$ have been measured in this alloy. The combination of these large 
$\Delta T_{\text {rev }}$ with a relatively large $\Delta S$ together with moderate elastic work confers an outstanding $C R P(\sim 2)$ to this $\mathrm{Ni}-\mathrm{Mn}$-Ti alloy. The superior mechanical properties of the alloy enable application of large $(\sim 1 \mathrm{GPa})$ stresses which anticipate a very large operational temperature window and long life fatigue.

As already mentioned, most magnetocaloric and electrocaloric materials also show giant mechanocaloric effects resulting from the interplay between magnetic or polar degrees of freedom and structure. Therefore, these materials show a cross-response to multiple external stimuli with associated multicaloric properties. The use of more than one driving field is interesting because it may reduce the field magnitude to achieve larger thermal effects and also may enlarge the operational temperature window. It also provides a strategy to improve the reversibility of a caloric effect by an effective control of the hysteresis. ${ }^{9,40}$ In addition, a suitable combination of magnetic and mechanical fields enables caloric cycles to be designed, which take advantage of hysteresis to reduce the volume of the magnetic field source needed to run the cycle. ${ }^{41}$

The excellent caloric performances discussed above for mechanocaloric materials open up encouraging prospects for their technological applications in heat pumping and refrigeration. Devices based on barocaloric effects have not been developed yet although designing and building challenges of a barocaloric cooler have been discussed in Ref. 42. More appealing is the elastocaloric effect for which several prototypes and demonstrators have already been proposed based on $\mathrm{Ni}$-Ti alloys. ${ }^{43-46}$ We believe that in a near future elastocaloric devices will improve the performances of present-day systems based on magetocaloric materials (which have been studied for many years). On the one hand, elastocaloric materials are, in general, formed by elements which are non-critical, cheap, abundant, and non-toxic. They often have thermal conductivities larger than magnetocaloric materials, which thus facilitates heat exchanges. On the other hand, elastocaloric devices are easily scalable and actually the proposed prototypes range from miniature ${ }^{47}$ to large scales. ${ }^{46}$ An advantage lies in the different possibilities for applying forces. In addition to traditional mechanical methods (screw driven, dead loads, etc.), there are alternative strategies which have recently been proposed such as using a magnetostrictive material to generate a stress ${ }^{48}$ (which is driven by a low magnetic field of $\sim 0.1 \mathrm{~T}$ ), or combining two shape memory alloys where one of them is used to generate a thermally driven stress. ${ }^{49}$

To conclude this article, we would like to point out that the study of mechanocaloric effects and materials is still in its early stages. Nevertheless, the recent emergence of interesting families of mechanocaloric materials suggests that there is plenty of room for improvement in both materials design and optimization. Furthermore, the use of the multicaloric response can help in overcoming a number of single caloric effect handicaps arising from high driving fields, narrow operational window, and reduced reversibility. We envisage a bright future for elastocaloric materials both in miniature and large-scale devices, and we expect to find them in a number of everyday cooling and heat pumping applications within a few years.

We acknowledge financial support from CICyT (Spain), Project No. MAT2016-75823-R.

\section{REFERENCES}

1. Mañosa, A. Planes, and M. Acet, J. Mater. Chem. A 1, 4925 (2013).

${ }^{2}$ J. P. Joule, Philos. Trans. R. Soc. London 149, 91 (1859).
${ }^{3}$ L. Mañosa and A. Planes, J. Phys. D 51, 070201 (2018); and references therein. ${ }^{4}$ V. K. Pecharsky and K. A. Gschneidner, Phys. Rev. Lett. 78, 4494 (1997).

${ }^{5}$ A. S. Mischenko, Q. Zhang, J. F. Scott, R. W. Whatmore, and N. D. Mathur, Science 311, 1270 (2006).

${ }^{6}$ E. Bonnot, R. Romero, E. Vives, L. Mañosa, and A. Planes, Phys. Rev. Lett. 100, 125901 (2008).

${ }^{7}$ L. Mañosa, D. González-Alonso, A. Planes, E. Bonnot, M. Barrio, J. L. Tamarit, S. Aksoy, and M. Acet, Nat. Mater. 9, 478 (2010).

${ }^{8}$ I. Takeuchi and K. Sandeman, Phys. Today 62(12), 48 (2015).

${ }^{9}$ E. Stern-Taulats, T. Castán, L. Mañosa, A. Planes, N. D. Mathur, and X. Moya, MRS Bull. 43, 295 (2018)

${ }^{10}$ T. Gottschall, K. P. Skokov, M. Fries, A. Taubel, I. Radulov, F. Scheibel, D. Benke, S. Riegg, and O. Gutfleisch, Adv. Energy Mater. 9, 1901322 (2019).

${ }^{11}$ B. Nair, T. Usui, S. Crossley, S. Kurdi, G. G. Guzman-Verri, X. Moya, and S. Hirose, Nature 575, 468 (2019).

${ }^{12}$ W. Goetzler, R. Zogg, J. Young, and C. Johnson, Energy Savings Potential and $R D$ Opportunities for Non-Vapor-Compression HVAC Technologies (Navigant Consulting, Inc., U.S. Department of Energy, 2014).

${ }^{13}$ R. Wang, S. L. Fang, Y. C. Xiao, E. L. Gao, N. Jian, Y. W. Li, L. L. Mou, Y. A. Shen, W. B. Zhao, S. T. Li et al., Science 366, 216 (2019).

${ }^{14}$ L. Mañosa and A. Planes, Adv. Mater. 29, 1603607 (2017).

${ }^{15}$ S. P. Vallone, A. N. Tantillo, A. M. dos Santos, J. J. Molaison, R. Kulmaczewski, A. Chapoy, P. Ahmadi, M. A. Halcrow, and K. G. Sandeman, Adv. Mater. 31, 1807334 (2019).

${ }^{16}$ P. Lloveras, E. Stern-Taulats, M. Barrio, J. L. Tamarit, S. Crossley, W. Li, V. Pomjakushin, A. Planes, L. Mañosa, N. D. Mathur, and X. Moya, Nat. Commun. 6, 8801 (2015).

17J. M. Bermúdez, M. Sánchez-Andújar, S. Castro-Garcia, J. López-Beceiro, R. Artiaga, and M. S. Senaris-Rodríguez, Nat. Commun. 8, 15715 (2017).

${ }^{18}$ B. Li, Y. Kawakita, S. Ohira-Kawamura, T. Sugahara, H. Wang, J. F. Wang, Y. N. Chen, S. I. Kawaguchi, S. Kawaguchi, K. Ohara et al., Nature 567, 7749 (2019).

${ }^{19}$ P. Lloveras, A. Aznar, M. Barrio, P. Negrier, C. Popescu, A. Planes, L. Mañosa, E. Stern-Taulats, A. Avramenko, N. D. Mathur et al., Nat. Commun. 10, 1803 (2019).

${ }^{20}$ A. Aznar, P. Lloveras, M. Barrio, P. Negrier, A. Planes, L. Mañosa, N. D. Mathur, X. Moya, and J. L. Tamarit, J. Mater. Chem. A 8, 639 (2020).

${ }^{21}$ A. Aznar, P. Lloveras, M. Romanini, M. Barrio, J. L. Tamarit, C. Cazorla, D. Errandonea, N. D. Mathur, A. Planes, X. Moya, and L. Mañosa, Nat. Commun. 8, 1851 (2017).

22J. A. Gough, Mem. Lit. Philos. Soc. Manchester 1, 288 (1805).

${ }^{23}$ Z. Xie, G. Sebald, and D. Guyomar, Appl. Phys. Lett. 107, 081905 (2015).

${ }^{24}$ E. O. Usuda, W. Imamura, N. M. Bom, L. S. Paixão, and A. M. G. Carvalho, ACS Appl. Polym. Mater. 1, 1991 (2019).

${ }^{25}$ Y. Yoshida, K. Yuse, D. Guyomar, J. F. Capsal, and G. Sebald, Appl. Phys. Lett. 108, 242904 (2016)

${ }^{26}$ E. Vives, S. Burrows, R. E. Edwards, S. Dixon, L. Mañosa, A. Planes, and R. Romero, Appl. Phys. Lett. 98, 011902 (2011).

${ }^{27}$ J. Cui, Y. M. Wu, J. Muehlbauer, Y. H. Hwang, R. Radermacher, S. Fackler, M. Wuttig, and I. Takeuchi, Appl. Phys. Lett. 101, 073904 (2012).

${ }^{28}$ J. Tusek, K. Engelbrecht, L. P. Mikkelsen, and N. Pryds, J. Appl. Phys. 117, 124901 (2015).

${ }^{29}$ R. Millán-Solsona, E. Stern-Taulats, E. Vives, A. Planes, J. Sharma, A. K. Nayak, K. G. Suresh, and L. Mañosa, Appl. Phys. Lett. 105, 241901 (2014).

${ }^{30}$ Y. Li, D. Zhao, J. Liu, S. Qian, Z. Li, W. Gan, and X. Chen, ACS Appl. Mater. Interfaces 10, 25438 (2018).

${ }^{31}$ S. Nikitin, G. Myalikgulyev, M. P. Annaorazov, A. L. Tyurin, R. W. Myndyev, and S. A. Akopyan, Phys. Lett. A 171, 234 (1992).

${ }^{32}$ A. Gràcia-Condal, E. Stern-Taulats, A. Planes, and L. Mañosa, Phys. Rev. Mater. 2, 084413 (2018).

${ }^{33}$ D. Y. Cong, W. X. Xiong, A. Planes, Y. Ren, L. Mañosa, P. Y. Cao, Z. H. Nie, X. M. Sun, Z. Yang, X. F. Hong et al., Phys. Rev. Lett. 122, 255703 (2019)

${ }^{34}$ A. Aznar, A. Gràcia-Condal, A. Planes, P. Lloveras, M. Barrio, J. L. Tamarit, W. X. Xiong, D. Y. Cong, C. Popescu, and L. Mañosa, Phys. Rev. Mater. 3, 044406 (2019). 
${ }^{35}$ L. Mañosa, S. Jarque-Farnos, E. Vives, and A. Planes, Appl. Phys. Lett. 103, 211904 (2013).

${ }^{36}$ F. Xiao, T. Fukuda, T. Kakeshita, and X. Jin, Acta Mater. 87, 8 (2015).

${ }^{37}$ E. Brück, H. Yibole, and L. Zhang, Philos. Trans. R. Soc. A 374, 20150303 (2016).

${ }^{38}$ S. Crossley, N. D. Mathur, and X. Moya, AIP Adv. 5, 067153 (2015).

${ }^{39}$ This expression computes the minimum work needed to reversibly drive the elastocaloric effect, and the resulting value is similar to the work computed under the common assumption of recovery of the released mechanical work.

${ }^{40}$ J. Liu, T. Gottschall, K. P. Skokov, J. Moore, and O. Gutfleisch, Nat. Mater. 11, 620 (2012).

${ }^{41}$ T. Gottschall, A. Gràcia-Condal, M. Fries, A. Taubel, L. Pfeuffer, L. Mañosa, A. Planes, K. P. Skokov, and O. Gutfleisch, Nat. Mater. 17, 929 (2018).

${ }^{42}$ E. Stern-Taulats, A. D'Ammaro, A. Avramenko, A. Robinson, and X. Moya, "First steps towards barocaloric refrigeration," paper presented at the
International Conference on Caloric Coolig, Thermag VIII, Darmstadt (Germany), September 2018.

${ }^{43}$ H. Ossmer, F. Wendler, M. Gültig, F. Lamvrecht, S. Miyazaki, and M. Kohl, Smart. Mater. Struct. 25, 085037 (2016).

${ }^{44}$ S. X. Qian, Y. L. Geng, Y. Wang, J. Z. Ling, Y. H. Hwang, R. Radermacher, I. Takeuchi, and J. Cui, Int. J. Refrig. 64, 1 (2016).

${ }^{45}$ J. Tusek, K. Engelbrecht, D. Eriksen, S. Dall'Olio, J. Tusek, and N. Pryds, Nat. Energy 1, 16134 (2016).

${ }^{46}$ S. M. Kirsch, F. Welsch, N. Michaelis, M. Schmidt, A. Wieczorek, J. Frenzel, G. Eggeler, A. Schütze, and S. Seelecke, Energy Technol. 6, 1567 (2018).

${ }^{47}$ F. Bruederlin, L. Bumke, C. Chluba, H. Ossmer, E. Quandt, and M. Kohl, Energy Technol. 6, 1588 (2018).

${ }^{48}$ H. Hou, P. Finkel, M. Staruch, J. Cui, and I. Takeuchi, Nat. Commun. 9, 4075 (2018).

${ }^{49}$ S. Qian, Y. Wang, L. Yuan, and J. Yu, Energy 182, 881 (2019). 